\title{
Simulation of Fracture and Delamination in Layered Shells due to Blade Cutting
}

\author{
F. Confalonieri ${ }^{1}$ and U. Perego ${ }^{1^{*}}$ \\ ${ }^{1}$ Department of Civil and Environmental Engineering, Politecnico di Milano, \\ Piazza Leonardo da Vinci, 32, Milano, Italy \\ e-mail: federica.confalonieri@polimi.it; umberto.perego@polimi.it \\ *corresponding author
}

\begin{abstract}
A new isotropic damage cohesive model for the simulation of mixed-mode delamination is presented. The model is based on consideration of the interface internal friction, naturally leading to coupled opening and shear damage mechanisms. Mixed-mode fracture energy turns out to be a direct outcome of the model and does not require the definition of an empirical law, additional to pure Mode I and II fracture energies. The model has been developed to account for delamination processes promoted by blade cutting of carton packages.
\end{abstract}

Keywords: Mixed-mode delamination, cohesive model, internal friction, isotropic damage.

\section{Introduction}

The finite element simulation of cutting and delamination in carton packages is a complex problem, involving several nonlinearities, each one requiring the development of ad hoc numerical tools. The adopted computational strategy for the simulation of cutting, based on the use of "directional cohesive elements", has been described in (Pagani et al, 2015). This work will rather focus on the modelling of the delamination problem. Carton packages present a layered structure, consisting of paperboard, low-density polyethylene coatings, decor layers and possible layers of other materials, such as aluminum layers for food protection, so that delamination is a common failure mechanism, in particular in those regions where the cutting process is in progress. Although a large number of works devoted to the formulation of cohesive laws has been proposed in the literature, many of them are based on strong assumptions on the loading path and on the mixed-mode failure properties, or lack of thermodynamic consistency. Real-life delamination processes are indeed often characterized by mixed-mode loading conditions with varying mode ratio. Moreover, as demonstrated by several experimental works (see e.g. Benzeggagh and Kenane, 1996), the fracture energy significantly grows in passing from pure Mode I crack loading to pure Mode II.

A new isotropic damage cohesive model, specifically conceived for mixed-mode delamination, based on consideration of an internal friction mechanism, is formulated in this paper. Section 2 presents the proposed cohesive law, while some consistency tests are discussed 
in Section 3 to verify the consistency of the model under different loading paths. Finally, a numerical example is presented in Section 4.

\section{Mixed-mode delamination model}

The model, based on an isotropic damage formulation, is developed in a thermodynamic framework, which guarantees its consistency for any loading path. Let us introduce the free energy per unit surface $\Psi$ defined as:

$$
\Psi=\frac{1}{2} K\left(\left\langle\delta^{n}\right\rangle_{-}\right)^{2}+\frac{1}{2}(1-d) K\left(\left\langle\delta^{n}\right\rangle_{+}\right)^{2}+\frac{1}{2}(1-d) K\left(\delta^{s}\right)^{2}
$$

being $d$ the isotropic damage variable, $\mathrm{K}$ the elastic stiffness of the interface (assumed equal in pure Mode I and in pure Mode II crack loading conditions), $\delta^{n}$ and $\delta^{s}$ the normal the shear opening displacements in the local reference frame. The Macauley brackets \langle\rangle , denoting the negative and the positive part of the normal relative displacement, are introduced to account for the unilateral effect. The static variables of the model are defined by the following set of state equations:

$$
\begin{gathered}
t^{n}=\frac{\partial \Psi}{\partial \delta^{n}}=K\left\langle\delta^{n}\right\rangle_{-}+(1-d) K\left\langle\delta^{n}\right\rangle_{+} \quad t^{s}=\frac{\partial \Psi}{\partial \delta^{s}}=(1-d) K \delta^{s} \\
Y=-\frac{\partial \Psi}{\partial d}=\frac{1}{2} K\left(\left\langle\delta^{n}\right\rangle_{+}\right)^{2}+\frac{1}{2} K\left(\delta^{s}\right)^{2}
\end{gathered}
$$

where $t^{n}$ and $t^{s}$ represent the cohesive tractions in the normal and shear directions respectively, and $Y$ is the strain energy released per unit growth of damage.

The interaction between normal and shear modes is governed by the definition of three damage modes, namely one opening- and two shear-dominated modes, in the plane of dimensionless cohesive tractions, as the ones shown in Fig. 1. Each damage mode is characterized by the normal unit vector $\mathbf{n}^{i}$. The inclination angle $\alpha$ of the two shear-dominated modes plays the role of a parameter of internal friction.

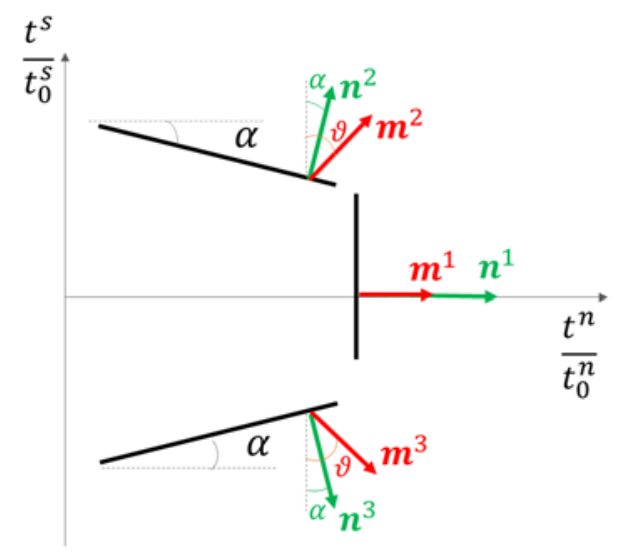

Fig. 1. Definition of damage mechanisms in tractions plane. 
In order to account for the contribution of every damage mode to the overall decohesion process, let us introduce the effective cohesive stresses $\mathbf{s}=\left[\mathrm{s}^{1} \mathrm{~s}^{2} \mathrm{~s}^{3}\right]^{\mathrm{T}}$, defined as the projection of the cohesive tractions onto the three normal vectors:

$$
\mathbf{s}=\mathbf{N} \overline{\mathbf{t}}
$$

where $\mathbf{N}$ is a matrix gathering the components of the three normal unit vectors, while $\overline{\mathbf{t}}$ is the vector collecting the dimensionless cohesive tractions in the local reference frame:

$$
\overline{\mathbf{t}}=\left[\begin{array}{ll}
\overline{t^{n}} & \overline{t^{S}}
\end{array}\right]^{T}=\left[\begin{array}{cc}
\frac{t^{n}}{t^{n}} & \frac{t^{s}}{t_{0}^{S}}
\end{array}\right]^{T} \quad \mathbf{N}=\left[\begin{array}{c}
\mathbf{n}^{1} \\
\mathbf{n}^{2} \\
\mathbf{n}^{3}
\end{array}\right]=\left[\begin{array}{cc}
1 & 0 \\
\sin \alpha & \cos \alpha \\
\sin \alpha & -\cos \alpha
\end{array}\right]
$$

being $t_{0}^{n}$ and $t_{0}^{S}$ the normal and the shear strengths in pure Mode I and II crack loading conditions. Writing eqn. (4) component-wise, one has:

$$
\begin{aligned}
& s^{1}=\overline{\mathbf{t}} \cdot \mathbf{n}^{1}=\bar{t} \\
& s^{2}=\overline{\mathbf{t}} \cdot \mathbf{n}^{2}=\overline{t^{n}} \sin \alpha+\overline{t^{s}} \cos \alpha \\
& s^{3}=\overline{\mathbf{t}} \cdot \mathbf{n}^{3}=\overline{t^{n}} \sin \alpha-\bar{t}^{s} \cos \alpha
\end{aligned}
$$

Similarly, the effective opening displacements, collected in the vector $\mathbf{w}=\left[\begin{array}{lll}w^{1} & w^{2} & w^{3}\end{array}\right]^{\mathrm{T}}$, can be defined as the projection of the dimensionless relative displacement $\bar{\delta}$ onto a structural unit vector $\mathbf{m}^{i}$. In matrix form:

$$
\mathbf{w}=\mathbf{M} \overline{\boldsymbol{\delta}}
$$

where $\mathbf{M}$ is a matrix gathering the structural unit vectors $\mathbf{m}^{\mathrm{i}}$, while $\overline{\boldsymbol{\delta}}$ is the vector collecting the dimensionless opening displacements in the local reference frame:

$$
\overline{\boldsymbol{\delta}}=\left[\begin{array}{ll}
\overline{\delta^{n}} & \overline{\delta^{s}}
\end{array}\right]^{T}=\left[\begin{array}{ll}
\frac{\delta^{n}}{\delta_{0}^{n}} & \frac{\delta^{S}}{\delta_{0}^{s}}
\end{array}\right]^{T} \quad \mathbf{M}=\left[\begin{array}{l}
\mathbf{m}^{1} \\
\mathbf{m}^{2} \\
\mathbf{m}^{3}
\end{array}\right]=\left[\begin{array}{cc}
a & 0 \\
b \sin \vartheta & b \cos \vartheta \\
b \sin \vartheta & -b \cos \vartheta
\end{array}\right]
$$

being $\delta_{0}^{n}$ and $\delta_{0}^{s}$ the opening displacements at the onset of delamination in pure Mode I and II crack loading conditions, respectively, corresponding to $t_{0}^{n}$ and $t_{0}^{s}$, and $\vartheta$ the angle defining the orientation of $\mathbf{m}^{2}$ and $\mathbf{m}^{3}$ ( $\mathbf{m}^{1}$ is directed as $\mathbf{n}^{1}$ for symmetry reasons). The constants $a$ and $b$ can be computed by imposing that the elastic strain energy is left unchanged, passing from the direct to the effective quantities, i.e.

$$
\frac{1}{2} \mathbf{t}^{T} \boldsymbol{\delta}=\frac{1}{2}\left(s^{1} w^{1}+s^{2} w^{2}+s^{3} w^{3}\right)
$$

From eqn. (9), one obtains:

$$
a=\left(t_{0}^{n} \delta_{0}^{0}-t_{0}^{S} \delta_{0}^{S} \tan \alpha \tan \vartheta\right) \quad b=\frac{t_{0}^{S} \delta_{0}^{S}}{2 \cos \alpha \cos \vartheta}
$$

Thus, Eq. (7) can be re-written as: 


$$
\begin{aligned}
& w^{1}=\overline{\boldsymbol{\delta}} \cdot \mathbf{m}^{1}=\left(t_{0}^{n} \delta_{0}^{n}-t_{0}^{s} \delta_{0}^{s} \tan \alpha \tan \vartheta\right) \overline{\delta^{n}} \\
& w^{2}=\overline{\boldsymbol{\delta}} \cdot \mathbf{m}^{2}=\frac{t_{0}^{s} \delta_{0}^{S}}{2 \cos \alpha \cos \vartheta}\left(\sin \vartheta \overline{\delta^{n}}+\cos \vartheta \overline{\delta^{s}}\right) \\
& w^{3}=\overline{\boldsymbol{\delta}} \cdot \mathbf{m}^{3}=\frac{t_{0}^{s} \delta_{0}^{S}}{2 \cos \alpha \cos \vartheta}\left(\sin \vartheta \overline{\delta^{n}}-\cos \vartheta \overline{\delta^{s}}\right)
\end{aligned}
$$

Exploiting the definition of effective stresses and displacements, the overall elastic strain energy can be additively decomposed into the three contributions associated to the damage modes:

$$
\Psi=\underbrace{\frac{1}{2} s^{1} w^{1}}_{\Psi 1}+\underbrace{\frac{1}{2} s^{2} w^{2}}_{\Psi^{2}}+\underbrace{\frac{1}{2} s^{3} w^{3}}_{\Psi^{3}}=\frac{1}{2} \mathbf{t}^{\mathbf{T}}
$$

Defining the strain energy released per unit growth of damage $Y^{i}$ associated to each mode is thus straightforward. Note that $\overline{t^{n}}=\overline{\delta^{n}}$ and $\overline{t^{s}}=\overline{\delta^{s}}$ :

$$
\begin{gathered}
Y^{1}=\frac{\partial \Psi^{1}}{\partial d}=\frac{1}{2}\left(t_{0}^{n} \delta_{0}^{n}-t_{0}^{s} \delta_{0}^{s} \tan \alpha \tan \vartheta\right)\left(\overline{\delta^{n}}\right)^{2} \\
Y^{2}=\frac{\partial \Psi^{2}}{\partial d}=\frac{1}{4} t_{0}^{s} \delta_{0}^{S}\left[\tan \alpha \tan \vartheta\left(\overline{\delta^{n}}\right)^{2}+\left(\overline{\delta^{s}}\right)^{2}+(\tan \alpha+\tan \vartheta)\left(\overline{\delta^{n}}\right)\left(\overline{\delta^{s}}\right)\right] \\
Y^{3}=\frac{\partial \Psi^{3}}{\partial d}=\frac{1}{4} t_{0}^{s} \delta_{0}^{S}\left[\tan \alpha \tan \vartheta\left(\overline{\delta^{n}}\right)^{2}+\left(\overline{\delta^{s}}\right)^{2}-(\tan \alpha+\tan \vartheta)\left(\overline{\delta^{n}}\right)\left(\overline{\delta^{s}}\right)\right]
\end{gathered}
$$

Using eqns. (13), it easy to verify that $Y=Y^{1}+Y^{2}+Y^{3}$, where $Y$ is defined in eqn. (3). The angle $\vartheta$ is a parameter affecting the way the strain energy release rate $Y$ is decomposed into its component. Henceforth, it will be assumed:

$$
\tan \vartheta=\frac{t_{0}^{n} \delta_{0}^{n}}{t_{0}^{s} \delta_{0}^{s}} \tan \alpha,
$$

such that $Y^{1}$ is always positive for any positive value of the normal opening displacement, under the condition $\alpha<45^{\circ}$. Note that, according to this decomposition, either $Y^{2}$ or $Y^{3}$ can attain negative values, though their sum is obviously always positive.

The damage activation function is written as a classical energy criterion:

$$
\varphi=\left(\frac{Y^{1}}{\chi_{0}^{1}+\chi^{1}(d)}\right)^{k}+H\left(Y^{2}\right)\left(\frac{Y^{2}}{\chi_{0}^{2}+\chi^{2}(d)}\right)^{k}+H\left(Y^{3}\right)\left(\frac{Y^{3}}{\chi_{0}^{3}+\chi^{3}(d)}\right)^{k}-1
$$

where $\chi_{0}^{i}$ represents the initial value of the threshold for the $\mathrm{i}$-th damage mode, while $\chi^{\mathrm{i}}$ is an internal variable describing its evolution, such that $\chi^{i}(0)=0$. The Heaviside functions $\left(H\left(Y^{i}\right)=0\right.$ for $Y^{i} \leq 0, H\left(Y^{i}\right)=1$ for $\left.Y^{i}>0\right)$ are introduced in eqn. (15) to avoid negative contributions to damage activation by possibly negative values of $Y^{2}$ or $Y^{3}$. The damage growth is ruled by the following evolution law: 


$$
\begin{gathered}
\dot{d}=-\frac{\frac{\partial \varphi}{\partial \delta^{n}} \dot{\delta}^{n}+\frac{\partial \varphi}{\partial \delta^{s}} \dot{\delta}^{s}}{\frac{\partial \varphi}{\partial d}}= \\
=-\frac{\left(\frac{\partial \varphi}{\partial Y^{1}} \frac{\partial Y^{1}}{\partial \delta^{n}}+\frac{\partial \varphi}{\partial Y^{2}} \frac{\partial Y^{2}}{\partial \delta^{n}}+\frac{\partial \varphi}{\partial Y^{3}} \frac{\partial Y^{3}}{\partial \delta^{n}}\right) \dot{\delta}^{n}+\left(\frac{\partial \varphi}{\partial Y^{1}} \frac{\partial Y^{1}}{\partial \delta^{s}}+\frac{\partial \varphi}{\partial Y^{2}} \frac{\partial Y^{2}}{\partial \delta^{s}}+\frac{\partial \varphi}{\partial Y^{3}} \frac{\partial Y^{3}}{\partial \delta^{s}}\right) \dot{\delta}^{s}}{\frac{\partial \varphi}{\partial \chi^{1}} \frac{\partial \chi^{1}}{\partial d}+\frac{\partial \varphi}{\partial \chi^{2}} \frac{\partial \chi^{2}}{\partial d}+\frac{\partial \varphi}{\partial \chi^{3}} \frac{\partial \chi^{3}}{\partial d}}
\end{gathered}
$$

Finally, the following classical loading/unloading conditions hold:

$$
\varphi \leq 0 \quad \dot{d} \geq 0 \quad \varphi \dot{d}=0
$$

The shape of the cohesive law in the traction-opening displacement plane is governed by the internal variables $\chi^{i}$. The case of a bilinear cohesive law is considered here. The use of any other functional form of the $t^{i}-\delta^{i}$ curve is also possible. For the bilinear law, it is possible to define the expression of $\chi^{i}$ as a function of the damage variable $d$, such that a linear softening branch is obtained for pure Modes I and II crack loading (see Fig. 2).
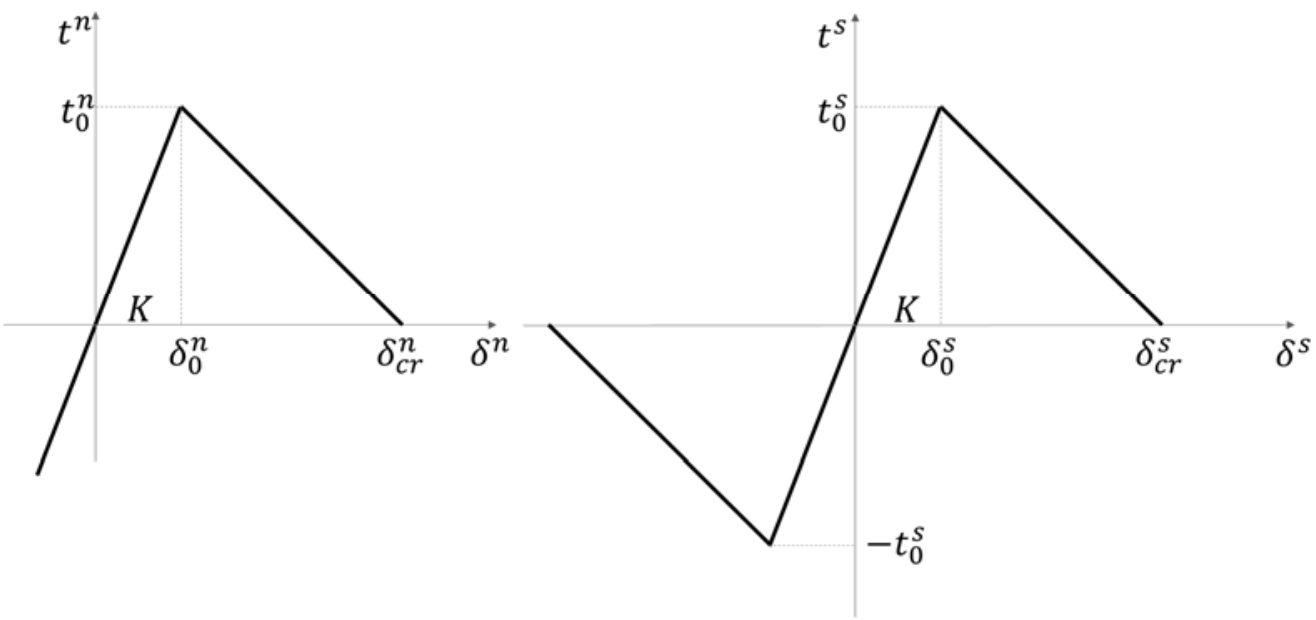

Fig. 2. Pure Mode I and II cohesive laws.

For the sake of simplicity, let us start from a 1D case, considering the bilinear law depicted in Fig. 3. The damage variable $d$ can be related to the current opening displacement $\delta$ by means of purely geometrical considerations, yielding:

$$
d=\frac{\delta_{c r}\left(\delta-\delta_{0}\right)}{\delta\left(\delta_{c r}-\delta_{0}\right)} \rightarrow \bar{\delta}=\frac{\delta_{c r}}{\delta_{c r}-\left(\delta_{c r}-\delta_{0}\right) d}
$$




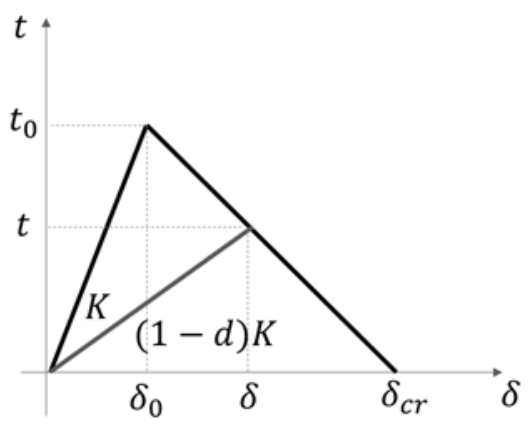

Fig. 3. Bilinear cohesive law.

In the $1 \mathrm{D}$ case, the damage activation function reduces to:

$$
\varphi=\left(\frac{Y}{\chi_{0}+\chi(d)}\right)^{k}-1
$$

being $Y=\frac{1}{2} K(\delta)^{2}=\frac{1}{2} t_{0} \delta_{0}(\bar{\delta})^{2}$. At the onset of delamination $\left(\delta=\delta_{0} \rightarrow \bar{\delta}=1\right)$, it holds that $d=0, \varphi=0$ and $\chi(0)=0$, therefore the initial threshold is simply obtained as:

$$
\left(\frac{\left.Y\right|_{\bar{\delta}=1}}{\chi_{0}}\right)^{k}=1 \quad \rightarrow \quad \chi_{0}=\left.Y\right|_{\bar{\delta}=1}=\frac{1}{2} t_{0} \delta_{0}
$$

while, in the softening phase:

$$
\left(\frac{Y}{\chi_{0}+\chi(d)}\right)^{k}=1 \quad \rightarrow \quad \chi=Y-\chi_{0}=\frac{1}{2} t_{0} \delta_{0}(\bar{\delta})^{2}-\frac{1}{2} t_{0} \delta_{0}
$$

By substituting eqn. (18) into eqn. (21), we get the expression of $\chi$ as a function of the damage variable $d$.

$$
\chi=\frac{1}{2} t_{0} \delta_{0}\left(\frac{\delta_{c r}}{\delta_{c r}-\left(\delta_{c r}-\delta_{0}\right) d}\right)^{2}-\frac{1}{2} t_{0} \delta_{0}
$$

Applying the same steps to each of the two pure Modes, the expressions for the three initial thresholds $\chi_{0}^{i}$ and for the static-like variables $\chi$ can be obtained. Note that, because of the symmetry of the activation domain, one has $\chi_{0}^{2}=\chi_{0}^{3}$ and $\chi^{2}=\chi^{3}$. In pure Mode II, $Y^{1}=0$, thus the Mode I dominated damage mode disappears from the damage activation function and $\chi_{0}^{2}=\chi_{0}^{3}$ and $\chi^{2}=\chi^{3}$ are independent of $\delta^{s}$. In contrast, in pure Mode I $Y^{2}$ and $Y^{3}$ are not zero, because of the coupling between normal and shear openings: as a consequence, $\chi_{0}^{1}$ and $\chi^{1}$ depend both on $\delta^{n}$ and $\delta^{s}$. The resulting expressions for the initial thresholds are:

$$
\chi_{0}^{1}=\frac{1}{\left[1-(\tan \alpha \tan \vartheta)^{k}\right]^{\frac{1}{k}}} \frac{1}{2}\left(t_{0}^{n} \delta_{0}^{n}-t_{0}^{s} \delta_{0}^{S} \tan \alpha \tan \vartheta\right)
$$




$$
\chi_{0}^{2}=\chi_{0}^{3}=2^{\frac{1}{k}} \frac{1}{4} t_{0}^{s} \delta_{0}^{S}
$$

while for the internal variables:

$$
\begin{gathered}
\chi^{1}=\frac{\frac{1}{2}\left(t_{0}^{n} \delta_{0}^{n}-t_{0}^{s} \delta_{0}^{s} \tan \alpha \tan \vartheta\right)}{\left[1-\left[\left(\frac{\delta_{c r}^{n}}{\delta_{c r}^{s}}\right)^{2}\left(\frac{\delta_{c r}^{s}-\left(\delta_{c r}^{s}-\delta_{0}^{s}\right) d}{\delta_{c r}^{n}-\left(\delta_{c r}^{n}-\delta_{0}^{n}\right) d}\right)^{2} \tan \alpha \tan \vartheta\right)^{k}\right]^{\frac{1}{k}}\left(\frac{\delta_{c r}^{n}}{\delta_{c r}^{n}-\left(\delta_{c r}^{n}-\delta_{0}^{n}\right) d}\right)^{2}-\chi_{0}^{1}} \\
\chi^{2}=\chi^{3}=2^{\frac{1}{k}} \frac{1}{4} t_{0}^{s} \delta_{0}^{S}\left(\frac{\delta_{c r}^{s}}{\delta_{c r}^{s}-\left(\delta_{c r}^{s}-\delta_{0}^{s}\right) d}\right)^{2}-\chi_{0}^{2}
\end{gathered}
$$

Using a classical argument, based on the Clausius-Duhem inequality for isothermal processes, it is possible to prove that the mechanical dissipation is always non-negative, being:

$$
D=Y^{1} \dot{d}+Y^{2} \dot{d}+Y^{3} \dot{d}=\left(Y^{1}+Y^{2}+Y^{3}\right) \dot{d}=Y \dot{d} \geq 0
$$

For the definition of its parameters $\left(\delta_{0}^{n}, \delta_{0}^{s}, \delta_{c r}^{n}, \delta_{c r}^{s}, \alpha, k\right)$, the proposed cohesive model requires the fracture energies $G_{I c}$ and $G_{I I c}$ and the peak tractions $t_{0}^{n}$ and $t_{0}^{S}$ in pure Modes I and II crack loading conditions, in addition to the curve describing the evolution of fracture energy with the mode mixity ratio. All this information can be obtained by means of standard experimental tests, i.e. one Double Cantilever Beam (DCB) test for pure Mode I, one End Notch Flexure (ENF) test for pure Mode II and a set of Mixed Mode Bending (MMB) tests (Reeder and Crews 1992) for varying mode-mixity ratio.

Figure 4a shows the initial activation surface $\varphi=0$, computed from eq. (15) for $d=0$, i.e. at the onset of decohesion, for increasing values of the internal friction angle $\alpha$ and for $k=2$. Conversely, Figure $4 \mathrm{~b}$ assesses the effect of increasing the exponent $\mathrm{k}$ while keeping a constant value of the angle $\alpha=30^{\circ}$. 

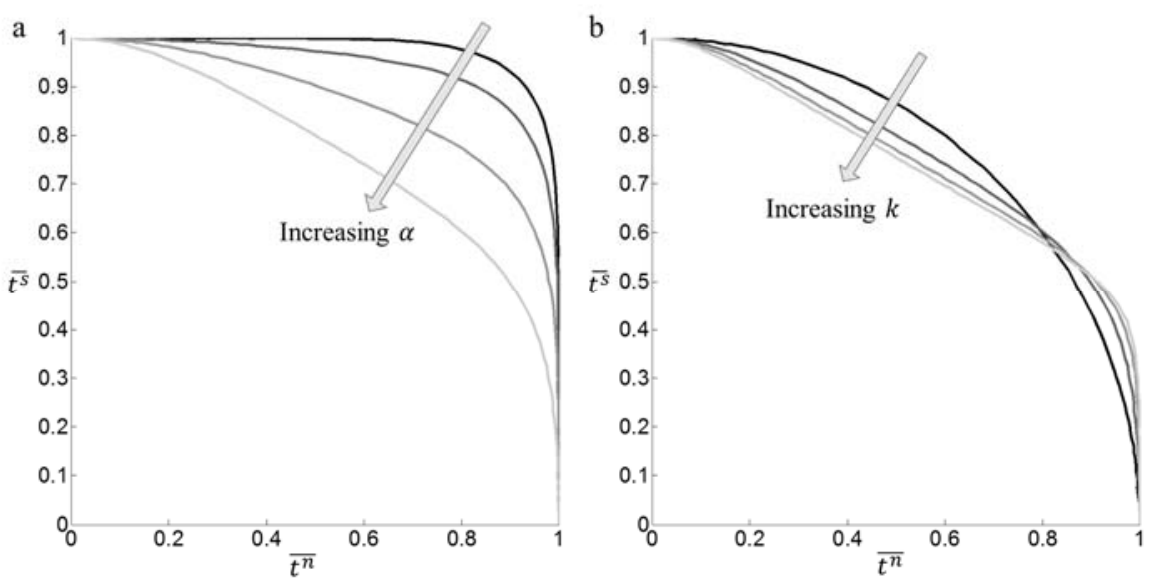

Fig. 4. a) Initial activation surface for $k=2$ and for increasing values of the angle $\alpha$ (namely $0^{\circ}, 10^{\circ}, 20^{\circ}$ and $30^{\circ}$ ). b) Initial activation surface for $\alpha=30^{\circ}$ and for increasing values of the exponent $k$ (namely $2,4,6,8$ ).

\section{Consistency tests}

Three different tests proposed in the literature are here considered to prove the consistency of the cohesive model, also in case of non-trivial loading paths.

\subsection{Radial path}

Radial loading conditions with varying separation angles can be achieved by imposing $\delta^{n}=$ $(1-\beta) \delta$ and $\delta^{s}=\beta \delta$, being $\delta$ an opening displacement linearly increasing from 0 to $\delta_{\max }=$ $0.05 \mathrm{~mm}$ and $\beta$ a coefficient describing the mode-mixity. Pure Mode I is recovered for $\beta=0$, while pure Mode II corresponds to $\beta=1$. The considered cohesive law properties for pure Mode I and II are reported in Table1.

\begin{tabular}{|c|c|c|c|c|}
\hline$t_{0}^{n}$ & $t_{0}^{S}$ & $G_{I c}$ & $G_{I I c}$ & $K$ \\
\hline $6 \mathrm{MPa}$ & $6 \mathrm{MPa}$ & $0.1 \mathrm{~N} / \mathrm{mm}$ & $0.1 \mathrm{~N} / \mathrm{mm}$ & $1000 \mathrm{~N} / \mathrm{mm}^{3}$ \\
\hline
\end{tabular}

Table 1. Cohesive law properties in pure Mode I and II.

The traction-separation curves for increasing values of the mode-mixity ratio $\beta$ are plotted in Figure 5. As expected, under mixed-mode conditions, the curves in the plane $t^{n}-\delta^{n}$ monotonically decrease starting from the pure Mode I one, while the curves in the plane $t^{s}-$ $\delta^{s}$ increase up to the pure Mode II bilinear law. 

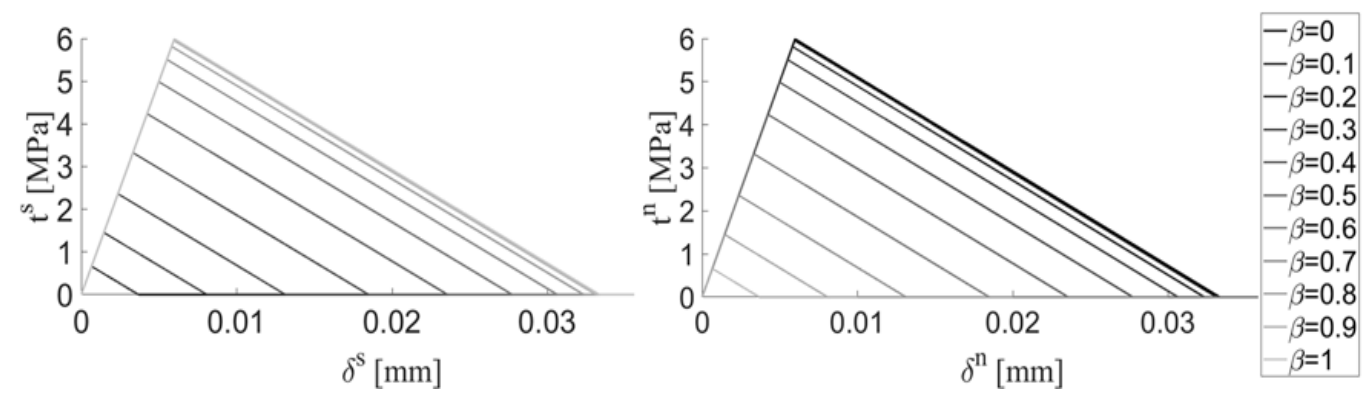

Fig. 5. Traction-separation curves for $\alpha=30^{\circ}$ and $k=2$.

\subsection{Sinusoidal path}

This consistency test has been proposed by Spring et al. (2016) to assess the consistency of the Park-Paulino-Roesler (PPR) cohesive model (Park et al, 2009) with respect to a mixed-mode loading/unloading condition. A sinusoidal history of opening displacements, displayed in Figure 6 , is considered here by assuming that $\delta^{n}=2 \cdot 10^{3} \mathrm{~mm} \sin \left(0.15 \mathrm{~s}^{-1} \tau\right)$ and $\delta^{n}=10^{3} \mathrm{~mm} 3.5 \sin \left(0.12 \mathrm{~s}^{-1} \tau\right)$, being $\tau$ a time-like parameter. The cohesive properties defined by Spring et al. (2016) under pure Mode I and pure Mode II crack loading conditions are listed in Table 2.

\begin{tabular}{|c|c|c|c|c|}
\hline$t_{0}^{n}$ & $t_{0}^{S}$ & $G_{I c}$ & $G_{I I c}$ & $K$ \\
\hline $40 \mathrm{MPa}$ & $15 \mathrm{MPa}$ & $0.1 \mathrm{~N} / \mathrm{mm}$ & $0.1 \mathrm{~N} / \mathrm{mm}$ & $10000 \mathrm{~N} / \mathrm{mm}^{3}$ \\
\hline
\end{tabular}

Table 2. Cohesive properties in pure Mode I and II.

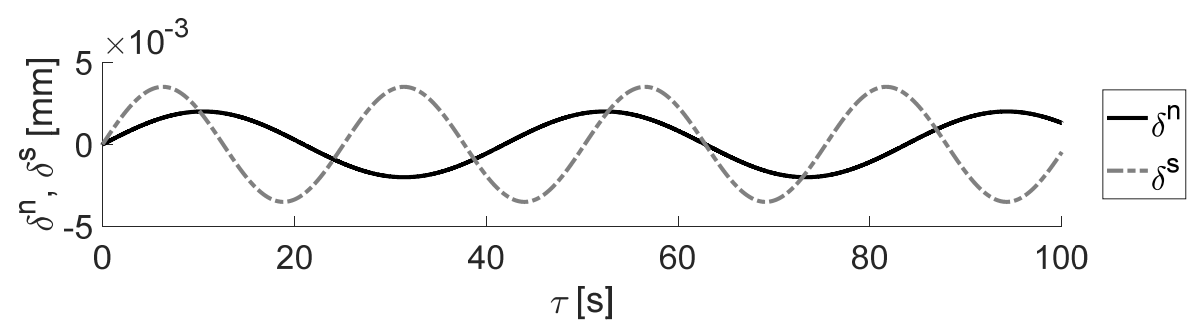

Fig. 6. History of applied normal and shear opening displacements.

Figure 7 shows the response of the cohesive model in terms of traction-separation curves, computed with $\alpha=30^{\circ}$ and $k=4$ As expected, during the loading/unloading phases, the damage does not increase and, thus, the slope remains constant. Note that in the case of a nonproportional loading path, although the pure Modes traction-separation curves are bi-linear, the resulting softening branch is not linear because of the coupling between normal and shear openings. 

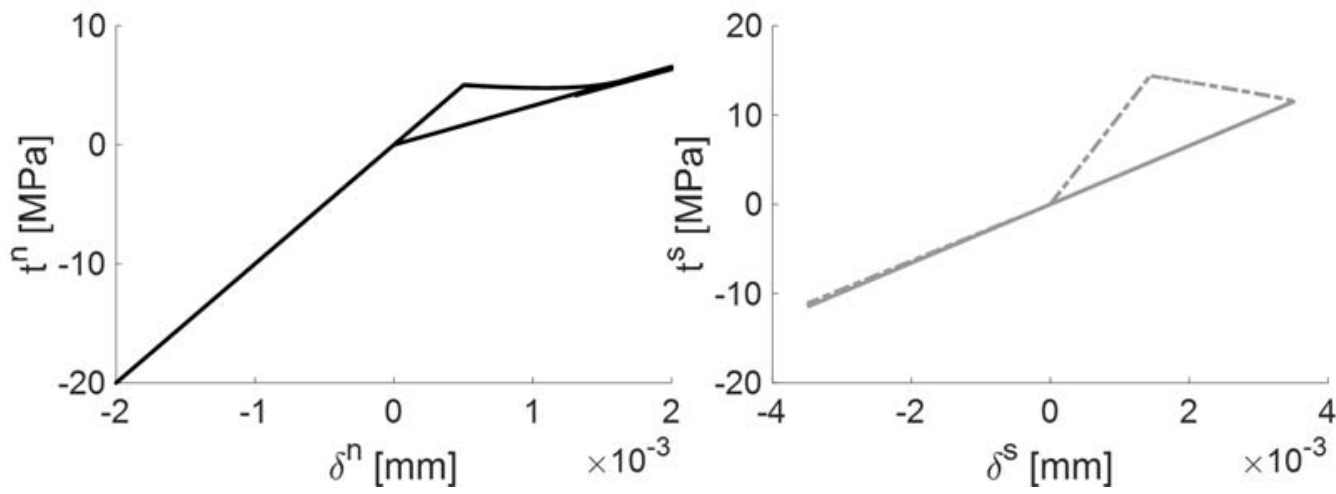

Fig. 7. Traction-separation curves for $\alpha=30^{\circ}$ and $k=2$, for sinusoidal path.

\subsection{Non-proportional loading path}

A non-proportional loading path, proposed by van den Bosch et al. (2006) and Park et al (2009) and depicted in Figure 8a, is considered here. The interface is loaded first in the normal direction up to $\delta^{n}=\Delta_{\max }^{n}$ and, then, in the shear direction up to failure, keeping the normal opening displacement constant. As in (van den Bosch et al. 2006) and (Park et al. 2009), consistency is tested in terms of the total work of separation, defined as:

$$
W=\underbrace{\int_{0}^{\Delta_{\max }^{n}} t^{n} d \delta^{n}}_{W^{n}}+\underbrace{\int_{0}^{\delta_{\max }^{s}} t^{s} d \delta^{s}}_{W^{s}}
$$

Table 3 summarizes the considered cohesive properties: the pure Modes are characterized by different values of strengths and fracture energy.

\begin{tabular}{|c|c|c|c|c|}
\hline$t_{0}^{n}$ & $t_{0}^{S}$ & $G_{I c}$ & $G_{\text {IIc }}$ & $K$ \\
\hline $6 \mathrm{MPa}$ & $12 \mathrm{MPa}$ & $0.1 \mathrm{~N} / \mathrm{mm}$ & $0.2 \mathrm{~N} / \mathrm{mm}$ & $10000 \mathrm{~N} / \mathrm{mm}^{3}$ \\
\hline
\end{tabular}

Table 3. Cohesive properties in pure Mode I and II.

Figure $8 \mathrm{~b}$ shows the works of separation computed for increasing values of $\Delta_{\text {max }}^{n}$, ranging from 0 to the value of complete decohesion. The proposed model is able to reproduce a smooth transition between the two values. 


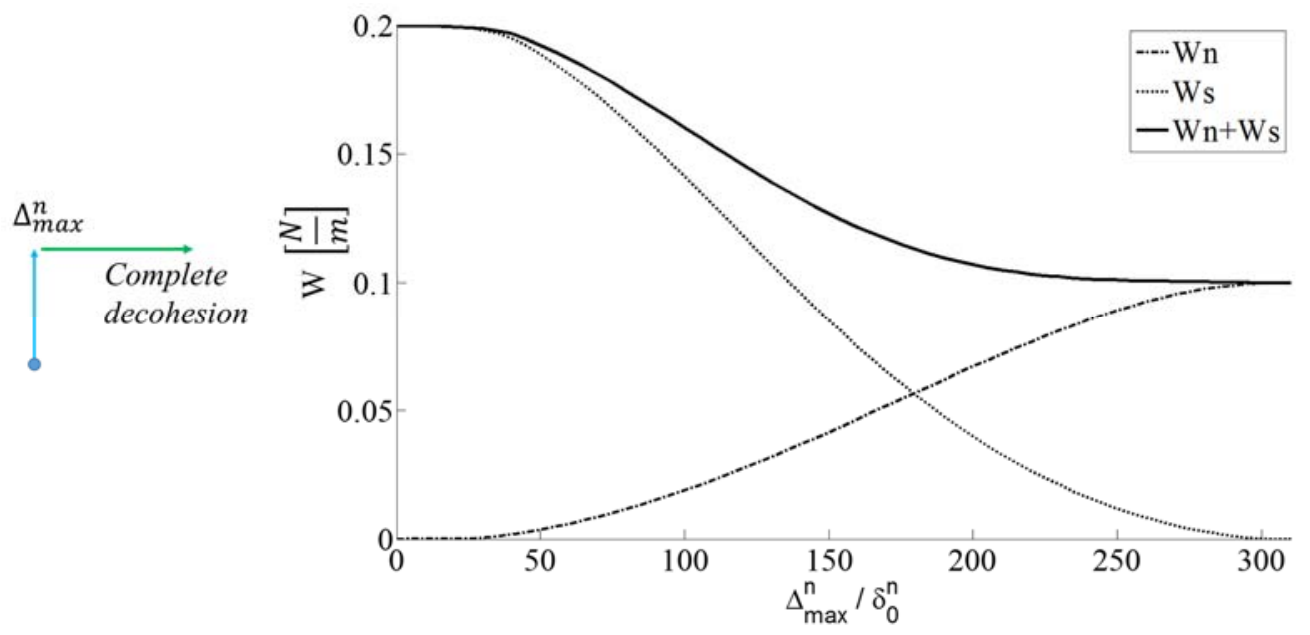

a)

b)

Fig. 8. a) Non-proportional loading path. b) Work of separation computed for $\alpha=30^{\circ}$ and $=$ 4 .

\section{Numerical example}

This numerical example assesses the capability of the proposed model to properly reproduce the mixed-mode behavior of a brittle epoxy resin (AS4/3501-6), experimentally tested through a set of MMB tests (Reeder and Crews, 1992) performed by Reeder (Reeder, 1993). The fracture energy $G_{c}=G_{I}+G_{I I}$ is computed as the sum of the areas $G_{I}$ and $G_{I I}$ beneath the normal and shear traction-separation curves, obtained with a series of radial paths with increasing moderatio. The adopted cohesive properties are: $G_{I c}=0.09 \mathrm{~N} / \mathrm{mm}, G_{I I c}=0.6 \mathrm{~N} / \mathrm{mm}, t_{0}^{n}=$ $45 \mathrm{MPa}, t_{0}^{n}=48 \mathrm{MPa}, \alpha=25^{\circ}$ and $k=12$. In figure 9 , the numerical prediction of the fracture energy for varying mode-mixity ratio is compared with the experimental data and with a Power Law, whose exponents have been calibrated in (Reeder, 1993). A very good agreement is obtained with the best fitting proposed by Reeder. It is worth underlining that the fracture energy is here an outcome of the model, without the need of introducing any empirical law for its variation with the mode-mixity ratio. 


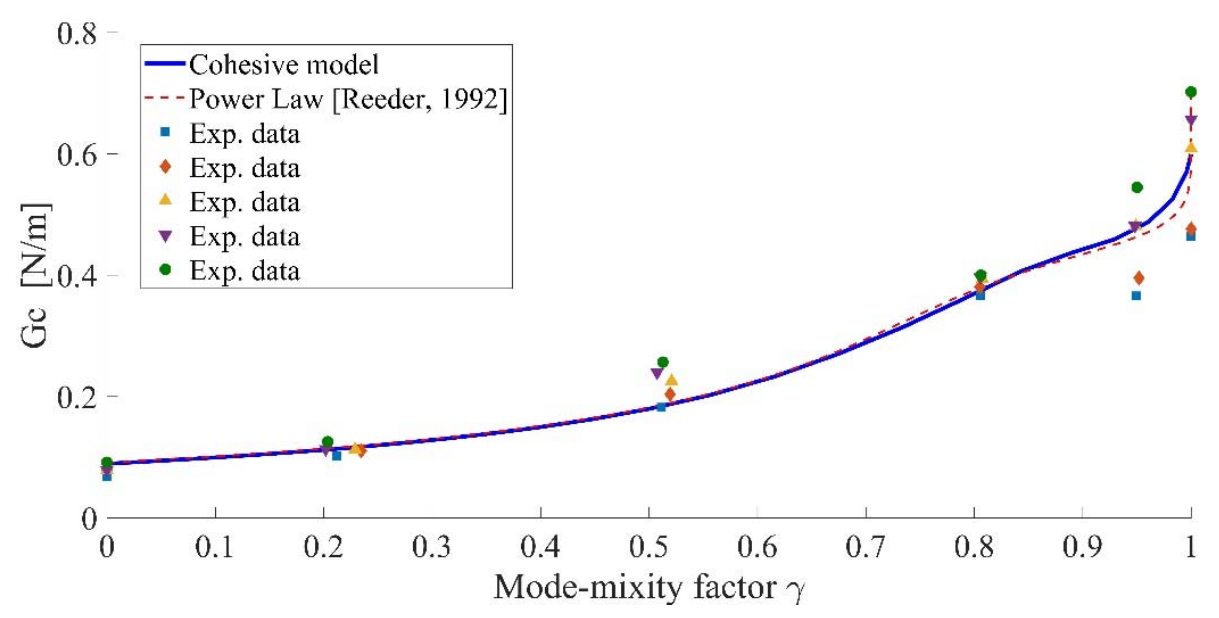

Fig. 9. Experimental and simulated mixed-mode fracture energies $\left(\alpha=25^{\circ}, k=12\right)$ for $\mathrm{MMB}$ test.

\section{Conclusions}

A new isotropic damage cohesive model has been proposed for the simulation of delamination under arbitrary, non-proportional mixed-mode loading conditions. The model is based on consideration of an internal friction dissipation mechanism, naturally resulting in a coupling between normal and shear damage modes. Tuning the internal friction parameter, it is possible to reproduce accurately the fracture energy under mixed-mode loading conditions, without introducing any empirical law to describe the variation of the fracture energy with the modemixity ratio. The model is thermodynamically consistent, as it implies rigorously positive dissipation, and exhibits a consistent mechanical response if used to simulate three widely employed consistency tests.

Acknowledgements The financial support of Tetra Pak Packaging Solutions is gratefully acknowledged.

\section{References}

Benzeggagh ML, Kenane M (1996). Measurement of mixed-mode delamination fracture toughness of unidirectional glass/epoxy composites with mixed-mode bending apparatus, Compos. Sci. and tech., 56, 439-449.

Pagani M, Perego U (2015). Explicit dynamics simulation of blade cutting of thin elastoplastic shells using "directional" cohesive elements in solid-shell finite element models. Computer Methods in Applied Mechanics and Engineering, 285, 515-541.

Park K, Paulino GH, Roesler JR (2009). A unified potential-based cohesive model of mixedmode fracture. Journal of the Mechanics and Physics of Solids, 57, 891-908.

Reeder JR, Crews JH Jr (1992). Redesign of the mixed-mode bending delamination test to reduce nonlinear effects, J. Compos. Tech. Res., 14, 12-19.

Reeder JR (1993). A bilinear failure criterion for mixed-mode delamination, Composite Materials, 
Spring D, Giraldo-Londono O, Paulino GH (2016). A study on the thermodynamic consistency of the Park-Paulino-Roesler (PPR) cohesive fracture model. Mechanics Research Communications, 78, 100-109.

Van den Bosch MJ, Schreurs PJG, Geers MGD (2006). An improved description of the exponential $\mathrm{Xu}$ and Needleman cohesive zone law for mixed-mode decohesion. Engineering Fracture Mechanics, 73, 1220-1234. 